\title{
Application of the Böhm chamber \\ for reference beta dose measurements and the calibration of personal dosimeters
}

Krystian Skubacz

\begin{abstract}
Thermoluminescent dosimeters (TLDs) currently used in personal and area dosimetry are often utilized to measure doses of ionizing radiation in fields with a more complex structure and therefore they should be calibrated in relation to different radiation types. The results of such calibration presented for UD-813 TLDs allowed for evaluation of their capability in relation to different radiation types like the beta and photon radiation of different energies and neutron radiation generated by the ${ }^{241} \mathrm{Am}-\mathrm{Be}$ source. The detector response for $60 \mathrm{keV}$ photons was $10 \%$ higher than for the $662 \mathrm{keV}$ gamma radiation of ${ }^{137} \mathrm{Cs}$. There were also response differences in relation to photon and beta radiation between detectors with an enhanced concentration of lithium ${ }^{6} \mathrm{Li}$ and boron ${ }^{10} \mathrm{~B}$ and detectors containing a natural level of these isotopes. Measurements of the reference beta doses were performed with the help of the Böhm chamber. This method is relatively more complicated compared to determining the reference photon and neutron doses and is described thoroughly in this paper. The corrected current measured by the Böhm chamber for the chosen parameters was a linear function for an entire available range of the chamber depths. The percentage of errors related to the evaluated reference beta doses were below $2 \%$ despite a rather large number of corrections that should be taken into account. The calibration distances varied from $11 \mathrm{~cm}$ to $50 \mathrm{~cm}$. For this range and beta particle energy, the absorption of radiation in the air was negligible and their attenuation had a predominantly geometric character.
\end{abstract}

Key words: beta radiation $・$ Böhm chamber $\bullet$ mixed fields $\bullet$ Panasonic TLDs

\section{K. Skubacz}

Silesian Centre for Environmental Radioactivity (BCR), Central Mining Institute,

1 Gwarków Sq., 40-166 Katowice, Poland,

Tel.: +48 32259 2816, Fax: +48 32259 2295,

E-mail: kskubacz@gig.eu

Received: 20 January 2015

Accepted: 16 November 2015

\section{Introduction}

The appropriate evaluation of measured doses in mixed fields requires the calibration of dosimeters in relation to different types of radiation and exposure conditions in order to split the signal into individual components corresponding to the specific radiation and exposure conditions of the entire period and evaluate the critical level, lower limit of detection and corresponding uncertainty [1]. The Panasonic UD-813 thermoluminescent dosimeters (TLDs) are used to evaluate mainly the doses related to neutrons but their design is appropriate for their use in the mixed field. Therefore, the dosimeters have to be irradiated to fulfil these tasks by beta, gamma, neutron and X-ray using the slab phantom or free in the air for environmental dosimetry.

The ${ }^{137} \mathrm{Cs}$ gamma sources and ${ }^{137} \mathrm{Sr}$ beta sources that were used to fulfil the task were mounted on the IM6/M-2 and IB1/P installation, respectively, and the X-ray that could be modified by filters recommended by the ISO standard 4037-1:1996 [2] was generated by the XCS-320-ST/X-RAY CAL (320 kV) device. All of these installations, made by TEMA 
(Italy) and Canberra-Packard Central Europe, Austria, were remotely controlled. The reference doses of gamma radiation and X-rays were measured by LS-01 or LS-10 ionizing chambers (PTW, Germany), which were calibrated in an accredited laboratory, and only the irradiation of dosimeters with neutrons generated by the ${ }^{241} \mathrm{Am}-\mathrm{Be}$ source was performed outside, in the National Centre for Nuclear Research in Świerk (Poland).

The reference dose corresponding to the beta radiation was measured by Böhm chamber, type 23392, made by PTW (Germany). This method is relatively more complicated compared to methods for determining the reference photon and neutron doses and is described in detail in this paper. The advantages of using these chambers are that they do not need to be calibrated and the reference doses can be determined precisely. However, this method demands the use of many corrections and is time-consuming. The way of its application, evaluation of the correction factors and the reference beta dose are described in this paper. Such a chamber can also be used for low-energy photon ionizing radiation; the number of corrections is somewhat reduced in this case.

\section{UD-813 dosimeters}

The Silesian Centre for Environmental Radioactivity (BCR) is equipped with an automatic UD-7900 Panasonic reader designed for large-scale measurements using thermoluminescent dosimeters. Among the available dosimeters, many of them can be applied in radiation fields with a complex structure to simultaneously measure doses related to different types of radiation. The evaluation of the results, however, requires the calibration of dosimeters in relation to various radiation sources and under different exposure conditions to split the signal into individual components and calculate the corresponding doses. This is the case of the UD-813 dosimeters, which were constructed by the Panasonic company mainly for the measurement of neutron doses [3]. However, due to their construction, they may also be used to determine the doses related to gamma and beta radiation. The TLD dosimeters are widely used for personal and sometimes area dosimetry all over the world $[4,5]$ because they take long-term measurements, are lightweight, have a small size and operate without a power supply. However, the response of this type of dosimeter depends on the neutron energy spectrum, and therefore a field calibration of dosimeters should be conducted for the proper evaluation of doses [6]. Other disadvantages are related to the relationship between the neutron cross-sections in tissue and in the thermoluminescence materials as reported by Olko [7].

All four detectors in the UD-813 dosimeter are made of $\mathrm{Li}_{2} \mathrm{~B}_{4} \mathrm{O}_{7}$ thermoluminescence material, but two of them, E1 and E4 (Fig. 1), are enriched with lithium ${ }^{6} \mathrm{Li}$ and boron ${ }^{10} \mathrm{~B}$ isotopes that have a considerably larger cross-section for interaction with thermal neutrons in comparison to the isotopes of lithium ${ }^{7} \mathrm{Li}$ and boron ${ }^{11} \mathrm{~B}$ that predominantly contain

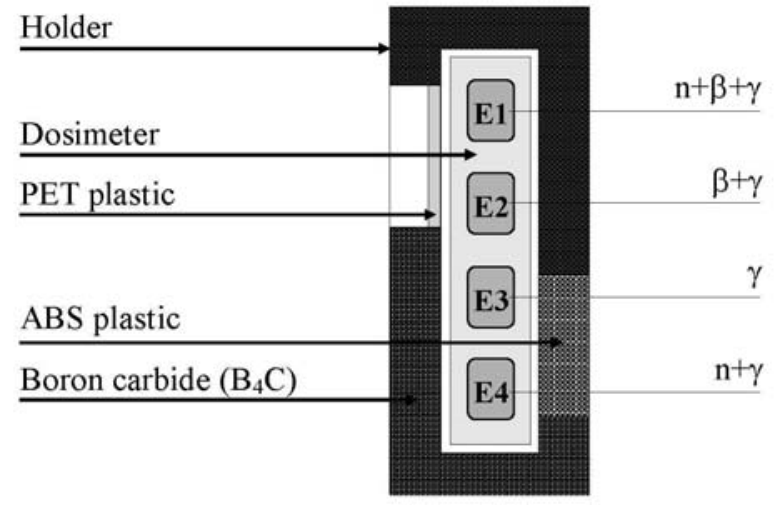

Fig. 1. Cross-section of the Wellhöfer holder with the UD-813 dosimeter inside.

naturally occurring materials (the cross-sections are about 26000 and 770000 times larger, respectively).

During the measurement, the dosimeter with four detectors is inside the Wellhöfer holder as is illustrated in Fig. 1. Because of the construction of this holder and the type of the thermoluminescence material, the detector E1 detects only the thermal neutrons that reach the dosimeter directly from the source. This is in contrast to the detector E4, which registers only the thermal neutrons that were moderated in the human body, called albedo neutrons $[3,8]$. This is possible thanks to a specially constructed holder that prevents detectors E1 and E4 from penetrating thermal neutrons from the back side and front side of the dosimeter, respectively. The non-thermal neutrons are detected with relatively poor efficiency. The neutron signal for both the E2 and E3 detectors can be neglected due to a low cross-section for neutron interaction. Beta radiation can be registered by the detectors E1 and E2 because the corresponding signal for E3 and E4 detectors is strongly reduced by the holder and dosimeter cover, and gamma radiation by all four detectors. Due to the location and type of the detectors in the holder and the filters applied, only gamma radiation is detected by the detector E3. As a result, the UD-813 dosimeters are useful to assess doses for personal or area dosimetry in mixed radiation fields. But, in order to get the appropriate results, the calibration factors and component signals associated with a particular type of radiation should be estimated. With an increasing number of interfering factors like background and different radiation types, the lower limit of detection also increases. Therefore, the quality of the dose evaluation depends to a large extent on the accurate calibration of dosimeters that should be conducted very carefully for different types of radiation and in conditions corresponding as closely as possible to the real exposure.

The following sections discuss how to perform such a calibration with respect to beta radiation with the help of the Böhm chamber. They also include the results of the calibration in relation to the neutron radiation of the ${ }^{241} \mathrm{Am}$-Be source, gamma radiation emitted by the ${ }^{137} \mathrm{Cs}$ isotope and the X-ray of the N3 narrow spectrum series corresponding to the ISO standard 4037-1:1996 [2]. 


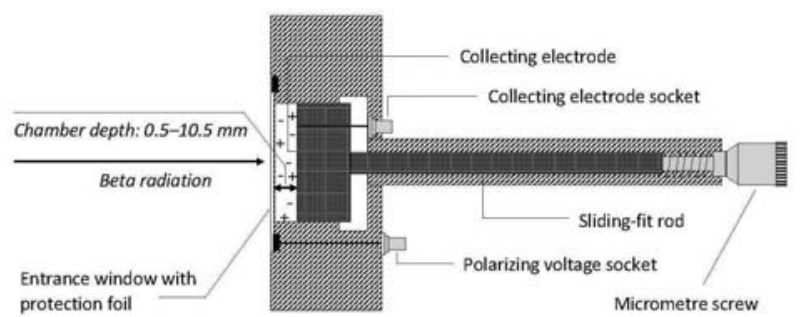

Fig. 2. Cross-section of the Böhm chamber.

\section{Böhm chamber}

\section{Principle of the measurement}

The Böhm chamber (Fig. 2) belongs to the group of so-called extrapolation ionization chambers that are recommended by the ISO standard 6980:2004 [9] to estimate the beta radiation dose for the calibration of dosimeters and dose-rate meters. The chamber was connected to the UNIDOS dosimeter (PTW, Freiburg). Such a configuration allowed for the assessment of beta radiation doses with energies exceeding $5 \mathrm{keV}$ and doses related to X-rays generated at a potential of more than $7.5 \mathrm{kV}$.

The evaluation of doses was carried out based on the assumption that the Bragg-Gray conditions were fulfilled, at which the ionization produced by radiation in a given small volume of air is related to the energy absorbed in the surrounding medium. Unless the beta radiation fluence is changed over this small volume, the dose or dose rate in a medium would be evaluated based on the quantity measured in the air. Therefore, the dose at the surface of a tissue is equal to

$$
\dot{D}_{T}(0)=\frac{S_{T}}{S_{A}} \dot{D}_{A}=\frac{S_{T}}{S_{A}} \frac{W}{e}\left(\frac{\partial I}{\partial m_{A}}\right)_{m_{A} \rightarrow 0}
$$

where $S_{T}$ and $S_{A}$ are the average mass collision stopping powers for tissue and air expressed as a ratio of the radiation energy lost while traversing a distance of $\partial x$ in a medium and the density of $\rho_{T}$ and $\rho_{A}$ for tissue and air, respectively. $W / e$ is the ratio of the average energy required to produce an ion pair in the air and its elementary charge, while $\left(\partial I / \partial m_{A}\right)_{m A \rightarrow 0}$ is the limiting value of the increment of the ionization current divided by the increment of air mass if the air mass $m_{A}$ tends to zero.

The Böhm chamber was developed to realize the Bragg-Gray conditions as closely as possible. The concept of small volume can be reached by changing the chamber depth step by step and by extrapolating the linear part of the $\partial I / \partial m_{A}$ function towards $x=0$. The slope of the linear part of the function can then be determined and, consequently, the dose rate $\dot{D}_{T}(0)$ can be calculated according to formula (1). The adjustment of the chamber depth is carried out manually with a micrometre screw in the range of $0.5 \mathrm{~mm}$ to $10.5 \mathrm{~mm}$ and with an accuracy of $1 \mu \mathrm{m}$.

In practice, radiation risk assessment is based on an evaluation of the personal dose equivalent $H_{p}(d)$ when applying individual dosimetry or the ambient dose equivalent $H^{*}(d)$ in the case of area monitor- ing. The first quantity is defined as an absorbed dose equivalent in a soft tissue below a specified point on the body at the appropriate depth $d$ while the ambient dose equivalent is the dose at a point in a radiation field that would be produced by the corresponding expanded and aligned field in a sphere with a $30 \mathrm{~cm}$ diameter of unit density tissue (ICRU sphere) at a depth $d$ on the radius vector opposing the direction of the aligned field [10]. The directional dose equivalent $H^{\prime}(d)$, which will be used further in this section, is equal to the ambient dose equivalent $H^{*}(d)$ if the angle between the radiation direction and a specified direction designated by the centre of the ICRU sphere and a point located at a depth $d$ is zero.

Doses that correspond to the beta radiation are determined mostly at the depth of $d=0.07 \mathrm{~mm}$ $\left(7 \mathrm{mg} / \mathrm{cm}^{2}\right)$. When assessing the dose equivalent for such a depth, the absorbed dose (1) should be multiplied by a so-called transmission coefficient $T(0.07)$ equal to $D_{T}(0.07) / D_{T}(0)$ and a conversion coefficient $K_{\text {DCF }}$ that converts the absorbed dose into the dose equivalent. This leads to the following relationship

$$
\dot{H}^{\prime}(0.07)=K_{\mathrm{DCF}} T(0.07) \frac{S_{T}}{S_{A}} \frac{W}{e}\left(\frac{\partial I}{\partial m_{A}}\right)_{m_{A} \rightarrow 0}
$$

The method of determining the dose with the Böhm chamber indicates that no calibration is necessary so that this method is an absolute one. However, because of the technical imperfections and deviations from the ideal Bragg-Gray conditions, Eq. (2) needs to be corrected. In addition, it is more convenient to perform the measurements for various chamber depths at the same saturation level of the current signal, and if this is not possible, then the appropriate corrections should be made. The electric field strength should not exceed $400 \mathrm{~V} / \mathrm{mm}$ because this could lead to uncontrolled changes of the chamber depth and, consequently, the corresponding current signal.

After the introduction of correction factors $k_{1}$ and $k_{2}$, the dose equivalent rate and its variance take the forms

$$
\begin{aligned}
& \dot{H}^{\prime}(0.07)=K_{\mathrm{DCF}} k_{1} T(0.07) \frac{S_{T}}{S_{A}} \frac{W}{e} \frac{1}{a \rho_{o}}\left(\frac{\partial k_{2} I}{\partial x}\right)_{x \rightarrow 0} \\
& \sigma_{H}^{2}=\dot{H}^{\prime}(0.07)\left(\begin{array}{c}
\frac{\sigma^{2}\left(k_{1}\right)}{k_{1}^{2}+\frac{\sigma^{2}(T(0.07))}{T^{2}(0.07)}} \\
+\frac{\sigma^{2}\left(\frac{S_{T}}{S_{A}}\right)}{\left(\frac{S_{T}}{S_{A}}\right)^{2}}+\frac{\sigma^{2}\left(\frac{W}{e}\right)}{\left(\frac{W}{e}\right)^{2}}+\frac{\sigma^{2}(a)}{a^{2}} \\
\left.+\frac{\sigma^{2}\left(\left(\frac{\partial k_{2} I}{\partial x}\right)_{x \rightarrow 0}\right)}{\left(\left(\frac{\partial k_{2} I}{\partial x}\right)_{x \rightarrow 0}^{2}\right.}\right)^{2}
\end{array}\right)
\end{aligned}
$$


The factor $k_{2}$ corrects the ionization current $I$, and finally the slope of the linear portion of the function $\partial I / \partial m_{A}$. The air mass $m_{A}$ of the measuring volume corresponding to the chamber depth was replaced in Eq. (3) by $m_{A}=x a \rho_{o}$, where $a$ is the effective collecting area and $\rho_{o}$ is the air density at the assumed reference conditions: absolute air temperature $T_{o}=293.15 \mathrm{~K}$ and air pressure $P_{o}=$ 1023.25 mbar. Additionally a relative humidity of $\mathrm{RH}_{o}=45 \%$ is considered as a reference value.

Another factor that changes the ionization current is the polarity current due to the absorption of beta particles behind the collecting electrode that diffuse to this electrode and socket connections, changing the polarity and resulting in the generation of an additional current. This polarity current is not caused by ionization, and an adequate correction should be made. The polarization effect is particularly pronounced for high-energy beta sources $\left({ }^{90} \mathrm{Sr} \mid{ }^{90} \mathrm{Y}\right.$ compared to ${ }^{204} \mathrm{Tl}$ or $\left.{ }^{147} \mathrm{Pm}\right)$, and its magnitude, at the given calibration distance, does not depend on the chamber depth and collecting potential [11]. The polarization current $I_{p}$ can be evaluated by measuring $I_{+}$and $I_{-}$currents at positive and negative polarities: $I_{p}=-\left(I_{+}+I_{-}\right) / 2$. Then, taking into account the collection efficiency $\Psi$, the corrected ionization current $I$ is equal to:

$$
\begin{aligned}
& I=\frac{I_{+}+\left|I_{P}\right|}{\Psi} \\
& \Psi=\left(1-\frac{2 k T}{e U}\right)\left(1-\frac{E x}{U}\right)\left(1-\frac{\Gamma x^{3}\left(I_{+}+\left|I_{P}\right|\right)}{a U^{2}}\right)
\end{aligned}
$$

where $U$ is the absolute value of the collecting voltage, $T$ is the air temperature, and $k$ is the Boltzmann constant. The remaining constants $E$ and $\Gamma$ are equal to $4.4 \mathrm{~V} \cdot \mathrm{m}^{-1}$ and $5.05 \cdot 10^{13} \mathrm{~V}^{2} \cdot \mathrm{A}^{-1} \cdot \mathrm{m}^{-1}$, respectively.

The factor $k_{1}$ is the product of four components: $k_{b r}, k_{b a}, k_{r a}$ and $k_{w i}$. The first one, $k_{b r}$, is related to the current $I_{b r}$ due to bremsstrahlung and can be evaluated by putting a poly (methyl methacrylate) (PMMA) absorber of $1 \mathrm{~cm}$ thickness in front of the entrance window. Such an absorber prevents the collecting volume of the Böhm chamber from the infiltration of beta particles, and the bremsstrahlung generated by its interaction with beta radiation can be neglected because of its low atomic number. A slightly different interaction of beta radiation with the PMMA in comparison to the tissue needs to be corrected by the second component $\left(k_{b a}\right)$. The third component, $k_{r a}$, takes into account the non-uniformity of the radiation beam in the plane perpendicular to its direction. The absorbed dose rate is averaged over the entire surface of the entrance window. Therefore, in cases where it is necessary to determine the dose value at the centreline of the beam in the place where the dosimeter will be located during calibration, the appropriate correction needs to be introduced unless the beam flattening filters according to ISO [9] have been applied. During our measurements, the beta radiation beam was not modified by such filters, and therefore the inhomogeneity of the beta radiation beam was evaluated using a matrix of thermoluminescent detectors. They were placed during irradiation at different points across a plane perpendicular to the radiation beam centreline, in the same position where the entrance window of the Böhm chamber was located during the reference dose rate measurements. The last component of the $k_{1}$ factor takes into account the attenuation of the beta radiation by the thin entrance window. It can be estimated by measurement of the current signal at the given value of the chamber depth for different PMMA absorbers that are located in front of the chamber. The PMMA surface mass needs to be multiplied by 0.920 to refer it to tissue [11]. The current value referring to the surface mass of the entrance window and the current $I(d=0)$ by extrapolation can be retrieved based on the functional dependence between the ionization current and surface mass of the absorbers. Moreover, the ratio $I(d) / I(0)$ can be regarded as a transmission coefficient $T(d)$ as long as the energy flux of beta radiation at the depth $d$ does not significantly affect the ratio of $S_{T} / S_{A}\left(k_{w i}\right)$. The aforementioned components were evaluated according to the following formulae:

$$
\begin{gathered}
k_{b r}=\frac{I-I_{b r}}{I}, k_{b a}=\frac{1}{B_{p}}\left(1+\frac{Z_{T}}{Z_{p}}\left(B_{p}-1\right)\right), \\
k_{r a}=\frac{M}{\sum_{i=1}^{M} \frac{R_{i}}{R_{C}}}
\end{gathered}
$$

where $Z_{T}$ and $Z_{p}$ are effective atomic numbers of the tissue and poly (methyl methacrylate) (PMMA) material respectively, $B_{p}$ is the backscatter coefficient of PMMA material, $R_{i}$ is the readout results of thermoluminescent detectors that were corrected for background and element correction factors, $R_{C}$ is the readout of the detector that was placed just on the beam centreline of beta radiation and $M$ is the number of irradiated detectors.

The second resultant correction factor, $k_{2}$, consists of eight components $k_{a d}, k_{a b}, k_{e l}, k_{i n}, k_{d i}, k_{p e}$, $k_{a c}$, and $k_{d e}$. The components that correspond to the attenuation of beta radiation due to absorption and scattering in the air between the radiation source and the entrance windows depending on air temperature, pressure, relative humidity $\left(k_{a b}\right)$, the uncontrolled change of the chamber depth as a result of electrostatic attraction $\left(k_{e l}\right)$, disturbance of the secondary electron flux because of different atomic numbers in the air of the collecting volume and entrance window or collecting electrode $\left(k_{i n}\right)$ and attenuation of the beta radiation caused by interaction with the air in the collecting volume $\left(k_{a c}\right)$, can be neglected for the ${ }^{90} \mathrm{Sr} \mid{ }^{90} \mathrm{Y}$ radiation source, conditions that are close to the reference conditions, applied chamber depths and collecting potential. Therefore, they are equal to 1 . Among the remaining components, the component reflecting the disturbance of the beta radiation flux caused by the side walls of the chamber $\left(k_{p e}\right)$ can be evaluated by measurements of the ionization current that are performed with the help of rings of different thicknesses and the same inner diameter located at the front of the entrance windows. The next component $\left(k_{a d}\right)$ is introduced to refer the air 
density inside of the collecting volume to the reference conditions. The last two components correct the divergence of beta radiation inside the collecting volume $\left(k_{d i}\right)$ and radioactive decay of the radiation source $\left(k_{d e}\right)$. All three of these can be estimated as follows [11]:

$$
k_{a d}=\frac{P_{o} T}{P T_{o}}, k_{d i}=1+\frac{x}{L}, k_{d e}=e^{-\frac{\Delta t \ln 2}{T_{1 / 2}}}
$$

where $P$ and $T$ are the air pressure and absolute temperature, respectively, when measuring the reference absorbed dose rate, $L$ is the calibration distance and $T_{1 / 2}$ is the half-life of the radiation source. If necessary, the correction for radioactive decay is taken into account to perform a retrospective evaluation of the measurement. This situation can occur when comparing the results of the two measurements made with the help of Böhm chambers at the $\Delta t$ time interval.

\section{Results}

The reference absorbed dose rates were determined in relation to the ${ }^{90} \mathrm{Sr}{ }^{90} \mathrm{Y}$ beta source at four calibration distances: $L=11,20,30$ and $50 \mathrm{~cm}$. This radiation source with the nominal activity of $50 \mathrm{mCi}$ at the reference date of 11 January 2011 is mounted on the remote-controlled IB1/P SP/BOT/R-2 installation (TEMA, Italy), which allows for the adjustment of the calibration distance to a maximum of $110 \mathrm{~cm}$. The Böhm chamber was supplied with a voltage of $150 \mathrm{~V}$ and the current was measured for the following chamber depths: $x=0.5,1,1.5,2$, 4, 6, 8 and $10 \mathrm{~mm}$. Table 1 shows the values of the parameters and components of the correction factor $k_{2}$ that were used to modify the current signal. The

Table 1. Parameters used to correct ionization current at a calibration distance of $30 \mathrm{~cm}$

\begin{tabular}{lc}
\hline \multicolumn{1}{c}{ Parameter } & Value \\
\hline$k_{a d}$ & $1.040 \pm 0.001$ \\
$k_{a b}$ & $1.000 \pm 0.000$ \\
$k_{e l}$ & $1.000 \pm 0.001$ \\
$k_{\text {in }}$ & $1.000 \pm 0.000$ \\
$k_{a c}$ & $1.000 \pm 0.000$ \\
$k_{d e}$ & $1.000 \pm 0.000$ \\
& From 1.002 to $1.033 \pm 0.000$ \\
$k_{d i}$ & to formula $(6)$ \\
& $0.997,0.993,0.990,0.986$ and \\
$k_{p e}$ & 2.0 mm and $x>2.0$ mm \\
& Freasing chamber depth $x$ according \\
$\Psi$ & chamber depth $x$ according to \\
& formula $(4)$ \\
$U[\mathrm{~V}]$ & $150.00 \pm 0.75$ \\
$\Gamma\left[\mathrm{V}^{2} \cdot \mathrm{A}^{-1} \cdot \mathrm{m}^{-1}\right]$ & $(5.05 \pm 0.25) \times 10^{13}$ \\
$E\left[\mathrm{~V} \cdot \mathrm{m}^{-1}\right]$ & 4.4 \\
$T[\mathrm{~K}]$ & $294.85 \pm 0.30$ \\
$a\left[\mathrm{~m}^{2}\right]$ & $7.2 \times 10^{-4} \pm 6.7 \times 10^{-7}$ \\
\hline
\end{tabular}

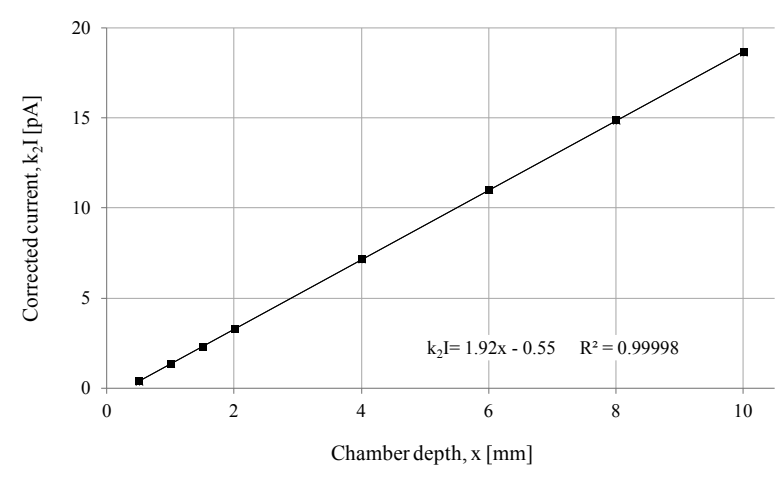

Fig. 3. Current signal as a function of the chamber depth at a calibration distance of $30 \mathrm{~cm}$.

component $k_{p e}$ was estimated by Böhm [11] for the ${ }^{90} \mathrm{Sr} \mid{ }^{90} \mathrm{Y}$ radiation source and the same ionization chamber was used for the current measurements.

The corrected ionization current at the calibration distance of $L=30 \mathrm{~cm}$ is almost a perfect linear function of the chamber depth with the correlation coefficient of $R=0.99998$ (Fig. 3). The correlation coefficients at the other calibration distances are also close to unity. The slopes of the line $\left(\partial k_{2} I / \partial x\right)_{x \rightarrow 0}$ were evaluated by linear regression with very good accuracy. For example, at the calibration distance of $L=30 \mathrm{~cm}$, its value was equal to $\left(1.924 \times 10^{-9}\right.$ $\left.\pm 3.146 \times 10^{-12}\right) \mathrm{A} \cdot \mathrm{m}^{-1}$.

The reference absorbed doses at all calibration distances have been estimated based on the calculated slopes of the lines. The value of parameters and particular components of the correction factors that were used in these calculations are presented in Table 2. The components of the correction factors $k_{b r}$ and $k_{w i}$ were adopted in accordance with Böhm [11], while others were calculated according to Eq. (5). The correction $k_{r a}$, related to the non-uniformity of the radiation beam in the plane perpendicular to its direction, was estimated with the help of UD-813 TLDs.

Table 2. Parameters used to evaluate the dose rate at a calibration distance of $30 \mathrm{~cm}$

\begin{tabular}{lc}
\hline \multicolumn{1}{c}{ Parameter } & Value \\
\hline$K_{\mathrm{DCF}}\left[\mathrm{Sv} \cdot \mathrm{Gy}{ }^{-1}\right]$ & 1 \\
$T(0.07)$ & $1.034 \pm 0.003$ \\
$S_{T} / S_{A}$ & $1.111 \pm 0.017$ \\
$W / e[\mathrm{~V}]$ & $33.97 \pm 0.05$ \\
$a\left[\mathrm{~m}^{2}\right]$ & $7.2 \times 10^{-4} \pm 6.7 \times 10^{-7}$ \\
$\rho_{o}\left[\mathrm{~kg} \cdot \mathrm{m}^{-3}\right]$ & 1.205 \\
$\left(\partial k_{2} I / \partial x\right)_{x \rightarrow 0}\left[\mathrm{~A} \cdot \mathrm{m}^{-1}\right]$ & $1.924 \times 10^{-9} \pm 3.146 \times 10^{-12}$ \\
$k_{b r}$ & $0.998 \pm 0.001$ \\
$k_{b a}$ & $1.010 \pm 0.000$ \\
$k_{r a}$ & $1.027 \pm 0.000$ \\
$k_{w i}$ & $0.997 \pm 0.002$ \\
$I_{P}[\mathrm{~A}]$ & $8.42 \times 10^{-14}$ \\
$B_{p}$ & $1.10 \pm 0.02$ \\
$Z_{p}$ & 5.85 \\
$Z_{T}$ & 6.50 \\
$P_{o}[\mathrm{mbar}]$ & 1013.25 \\
$P[\mathrm{mbar}]$ & 980 \\
$T_{o}[\mathrm{~K}]$ & 293.15 \\
$T[\mathrm{~K}]$ & $294.85 \pm 0.30$ \\
\hline
\end{tabular}




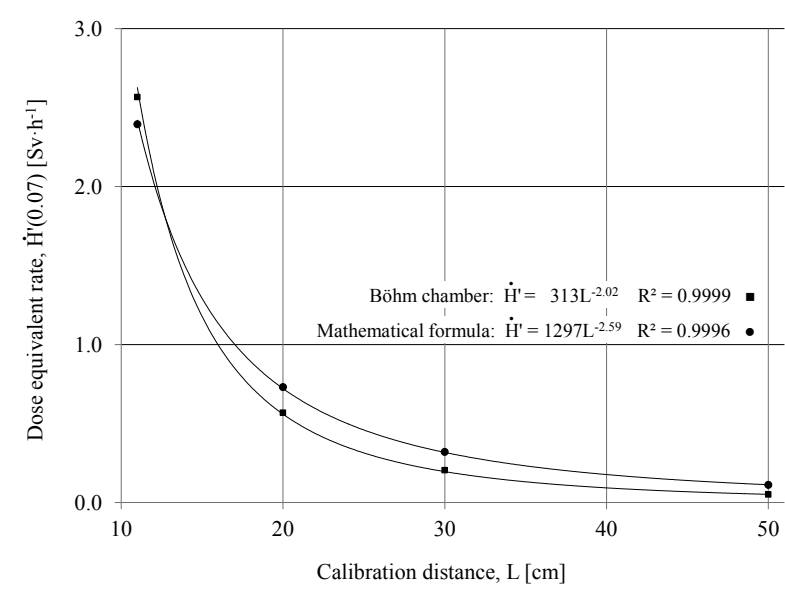

Fig. 4. The directional dose equivalent rate as a function of the calibration distance.

Figure 4 shows the functional dependence of the directional dose equivalent rate related to beta radiation and calibration distance. The function indicates that decreasing the dose equivalent rate is associated mainly with the geometric dispersion of the radiation flux while the absorption and scattering of beta particles in the air contribute only a little to the total attenuation of beta radiation. This is consistent with the observations made by Böhm [11], which state that the absorption of beta particles emitted by the ${ }^{90} \mathrm{Sr} \mid{ }^{90} \mathrm{Y}$ source is small at a calibration distance not exceeding $50 \mathrm{~cm}$ and under conditions close to the reference conditions $\left(P_{o}, T_{o}, \mathrm{RH}_{o}\right)$. In contrast, if the dose rate is assessed in accordance with the approximate formulae for the average energy of beta particles, their interaction with air has a much greater significance (Fig. 4).

Despite the large number of correction factors, the measurement of the absorbed dose rate with the help of the Böhm chamber can be made with good accuracy. The errors at the $1 \sigma$ confidence level were not greater than $1.8 \%$ (Table 3).

Table 3. The measured reference dose equivalent rates

\begin{tabular}{ccc}
\hline $\begin{array}{c}\text { Calibration } \\
\text { distance, } L \\
{[\mathrm{~cm}]}\end{array}$ & $\begin{array}{c}\text { Dose equivalent rate } \\
\dot{H}^{\prime}(0.07) \\
{\left[\mathrm{Sv} \cdot \mathrm{h}^{-1}\right]}\end{array}$ & $\begin{array}{c}\text { Percentage } \\
\text { error } \\
{[\%]}\end{array}$ \\
\hline 11 & $2.413 \pm 0.043$ & 1.8 \\
20 & $0.736 \pm 0.013$ & 1.8 \\
30 & $0.323 \pm 0.006$ & 1.8 \\
50 & $0.113 \pm 0.002$ & 1.8 \\
\hline
\end{tabular}

\section{Calibration of the UD-813 dosimeters}

As was previously mentioned, the UD-813 dosimeters are designed to measure doses of neutron, beta and photon radiation, and the calibration factors in relation to relevant detectors should be evaluated for all of these types of radiation. Because of the holder design, the signal due to beta radiation is strongly reduced for E3 and E4 detectors, and therefore the corresponding calibration factors were calculated for the E1 and E2 detectors. In the case of the albedo neutrons, the calibration factor was evaluated for the E4 detector and for the photons of X-ray and gamma radiation for all detectors. The calibration was performed by means of the UD-813 reference dosimeters that were chosen from the entire charge. These dosimeters were first irradiated in the National Centre for Nuclear Research in Świerk (Poland) by the ${ }^{241} \mathrm{Am}-\mathrm{Be}$ source with a reference neutron dose. The same dosimeters were then calibrated in the BCR laboratory in relation to the X-ray of the N3 narrow spectrum series corresponding to ISO [2], gamma radiation of cesium ${ }^{137} \mathrm{Cs}$ and beta radiation of strontium ${ }^{90} \mathrm{Sr} \mid{ }^{90} \mathrm{Y}$. The beta doses at the calibration installation were determined using the Böhm chamber.

The calibration factors and other quantities like uncertainties, critical levels and lower limits of detections were evaluated according to the developed algorithm [1]. The average results, which refer to the reference dosimeters, are presented in Table 4. During irradiation, they were located on the slab phantom to determine the calibration coefficients that correspond to the personal dose equivalents $H_{p}$. According to the test and the algorithm, the lower limit of detection in relation to ionizing photon radiation is equal to $0.1 \mathrm{mSv}$ at the significance level of $5 \%$, which is 100 times higher than for the most popular UD-802 dosimeters [12]. In relation to photon and beta radiation, the E2 and E3 detectors are slightly more sensitive than the $\mathrm{E} 1$ and $\mathrm{E} 4$ detectors.

The response of the detectors for $60 \mathrm{keV}$ and $662 \mathrm{keV}$ photon radiation can be expressed as the reciprocal of the calibration factor divided by the corresponding conversion coefficients. The results indicate that the response with respect to photon is only about $10 \%$ more sensitive for softer photon radiation. The results indicate that the sensitivity of the detectors with respect to photon and beta radiation is comparable, and they are only about $10 \%$ more sensitive to softer photon radiation. The detection capability is significantly worse in relation

Table 4. Averaged calibration coefficients $K_{X}, K_{G}, K_{\beta}$ and $K_{N}$

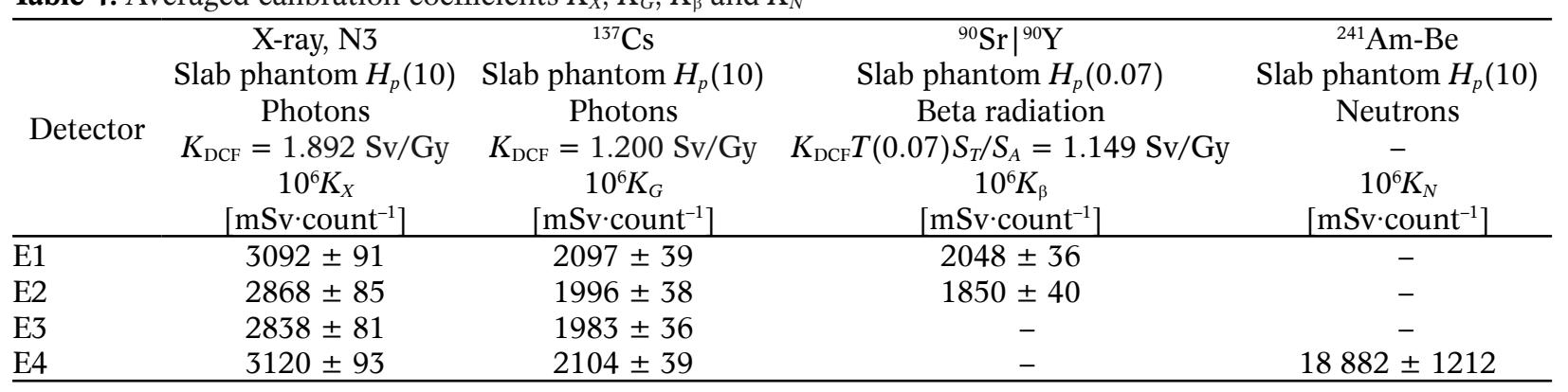


to neutron radiation, although the $\mathrm{E} 4$ detector is enriched with the isotopes of lithium ${ }^{6} \mathrm{Li}$ and boron ${ }^{10} \mathrm{~B}$ that have a relatively large cross-section for interaction with thermal neutrons.

\section{Conclusions}

The measurements of the reference dose with the Böhm chamber are quite labor-intensive and time-consuming. However, the chamber does not need to be calibrated, and such a method is recommended by the ISO standard 6980:2004 [9]. After taking into account the described correction factors, the results are not encumbered with a large uncertainty exceeding $2 \%$ at the $1 \sigma$ confidential level. Moreover, in comparison to other devices, it is possible to measure doses or dose rates in the relatively intense beta radiation fields.

The determination of the reference beta radiation doses enabled the calibration of the UD-813 dosimeters in relation to beta radiation and their application in the mixed radiation fields. Comparing the averaged calibration factors, the following conclusions can be drawn: the response for photon radiation with energies of about $60 \mathrm{keV}$ (X-ray, N3) is about $10 \%$ better than for higher energies of gamma radiation emitted by ${ }^{137} \mathrm{Cs}(662 \mathrm{keV})$. The dosimeters have much worse sensitivity in relation to neutron radiation.

\section{References}

1. Skubacz, K. (2015). Measurements of low doses with Panasonic dosimeters. Algorithm and tests. Radiat. Meas., 75, 9-14. DOI: 10.1016/j.radmeas.2015.02.020.

2. International Organization for Standardization. (1996). $\mathrm{X}$ and gamma reference radiation for calibrating dose- meters and doserate meters and for determining their response as a function of photon energy - Part 1: Radiation characteristics and production methods. ISO 4037-1-1996. Geneva.

3. Piesch, E., \& Burgkhardt, B. (1988a). Albedo dosimetry system for routine personnel monitoring. Radiat. Prot. Dosim., 23(1/4), 117-120.

4. Olko, P., Currivan, L., Van Dijk, J. W. E., Lopez, M. A., \& Wernli, C. (2006). Thermoluminescence detectors applied in individual monitoring of radiation workers in Europe - a review based on the EURADOS questionnaire. Radiat. Prot. Dosim., 120(1/4), 298-302. DOI: $10.1093 / \mathrm{rpd} / \mathrm{nci} 538$.

5. Rivera, T. (2012). Thermoluminescence in medical dosimetry. Appl. Radiat. Isot., 71, 30-34. DOI: 10.1016/j.apradiso.2012.04.018.

6. Piesch, E., \& Burgkhardt, B. (1988b). Field calibration technique for albedo neutron dosemeters. Radiat. Prot. Dosim., 23(1/4), 121-126.

7. Olko, P. (2010). Advantages and disadvantages of luminescence dosimetry. Radiat. Meas., 45(3/6), 506-511. DOI: 10.1016/j.radmeas.2010.01.016.

8. Budzanowski, M., \& Burgkhardt, B. (1995). Thin 6/7LiF'Mg,Cu,P and 6/7 LiF:Mg,Ti detectors for automatic albedo neutron dosimetry. Radiat. Meas., 24(4), 445-448. DOI: 10.1016/1350-4487(95)00015-7.

9. International Organization for Standardization. (2004). Nuclear energy - Reference beta-particle radiation - Part 2: Calibration fundamentals related to basic quantities characterizing the radiation field. ISO 6980-2:2004. Geneva.

10. International Commission on Radiation Units and Measurements. (1998). Conversion coefficients for use in radiological protection against external radiation. Bethesda: ICRU. (Report 57).

11. Böhm, J. (1986). The national primary standard of the PTB for realizing the unit of the absorbed dose rate to tissue for beta radiation. Braunschweig: Physikalish-Technische Bundesanstalt. (PTB-Dos-13).

12. Urban, P., \& Skubacz, K. (2013). Large-scale system for dosimetric measurements in industry and medicine. Zeszyty Naukowe WSZOP, 1(9), 68-82. 